\title{
THE CENTRALIZER OF THE GENERAL LINEAR GROUP
}

\author{
by C. J. MAXSON and A. OSWALD
}

(Received 25th May 1983)

\section{Introduction}

Let $G$ be a group, written additively with identity 0 , but not necessarily abelian and let $S$ be a semigroup of endomorphisms of $G$. The set $\mathscr{C}(S ; G)=\{f: G \rightarrow G \mid f \sigma=\sigma f$ for all $\sigma \in S$ and $f(0)=0\}$ is a zero-symmetric near-ring with identity under the operations of function addition and composition, called the centralizer near-ring determined by the pair $(S, G)$. Centralizer near-rings are general, for if $N$ is any zero-symmetric near-ring with identity then there exists a group $G$ and a semigroup $S \subseteq$ End $G$ such that $N \cong \mathscr{C}(S ; G)$. For background material and definitions relative to near-rings in general we refer the reader to the book by Pilz [7]. For material on centralizer near-rings we refer the reader to [4] and [6].

For $A$, a set of linear transformations on a vector space $V$ with certain conditions, the structure theory of the ring of linear transformations which commute with every element of $A$ has been investigated (e.g., [1], p. 32). In [2], the non-linear analogue for the case in which $V$ is a finite vector space and $A$ is generated by an invertible matrix is studied. This is extended in [4] to include the structure of $\mathscr{C}(A ; V)$ where $V$ is a finite vector space and $A \subseteq$ Aut $V$. For infinite $V$, the situation is much more difficult. The main structural results for $V$ infinite deal with the question of the simplicity of $\mathscr{C}(A ; V)$, $A \subseteq$ Aut $V$. (See [6] and [8].) It is thus the purpose of this paper to investigate the structure of $\mathscr{C}(\mathscr{U} ; V)$ where $V$ is an abelian group and $\mathscr{U}$ is the general linear group of size $n$ over a field $F$ with $\mathscr{U} \subseteq$ Aut $V$. This study then complements and extends the results in [2] and [4] as well as providing structural theory information about the infinite case.

Throughout this paper $\mathscr{U}$ will denote the general linear group $G L_{n}(F)$ of $n \times n$ matrices over a field $F$ where we always assume $n \geqq 2$, and $V$ will be an abelian group such that $\mathscr{U} \subseteq$ Aut $V$. Using the fact that the simple ring $R=M_{n}(F)$, i.e., the ring of $n \times n$ matrices over $F$, is generated by $\mathscr{U}$, the action of $\mathscr{U}$ on $V$ can be extended so that $V$ becomes a faithful, unital $R$-module. Since $R=R E_{11} \oplus \cdots \oplus R E_{n n}$ where the $E_{i i}$, $i=1,2, \ldots, n$, are the orthogonal idempotents $E_{i i}$ with 1 in position $(i, i)$ and 0 elsewhere, it follows that $V$ is the direct sum of irreducible $R$-modules, $V=\Sigma \oplus R E_{\alpha} m_{\alpha}$ where $E_{\alpha}$ is one of the idempotents $E_{i i}$ and $m_{\alpha} \in V$. If $E_{\alpha}=E_{i i}$, then the coefficients of $m_{\alpha}$ in $R E_{\alpha} m_{\alpha}$ 
are matrices with at most the ith column different from zero. In representing these elements we will often omit the zero columns and write

$$
\left[\begin{array}{c}
a_{1 i} \\
\vdots \\
a_{n i}
\end{array}\right] m_{\alpha} \text { for }\left[\begin{array}{ccc}
0 & a_{1 i} & 0 \\
\vdots & \vdots & \vdots \\
0 & a_{n i} & 0
\end{array}\right] m_{\alpha}
$$

We have therefore the situation in which $V$ is a unital $R$-module where $R$ is a simple ring contained in End $V$. Since $\mathscr{U} \subseteq R, \mathscr{C}(R ; V) \subseteq \mathscr{C}(\mathscr{U} ; V)$. The centralizer near-ring $\mathscr{C}(R ; V)$ where $V$ is a finite, faithful unital module over the finite simple ring $R$ has been the object of study in [3]. It was shown there that $\mathscr{C}(R ; V)$ is a simple near-ring, in fact a simple ring unless $R$ is a field and $\operatorname{dim}_{R} V>1$. The proof given in [3] also applies to the present situation where $R=M_{n}(F), F$ not necessarily finite, so here also one has that $\mathscr{C}(R ; V)$ is a simple near-ring and is a ring unless $R$ is a field and $\operatorname{dim}_{R} V>1$. One is thus lead to consider if these properties are inherited by $\mathscr{C}(\mathscr{U} ; V)$. Our work in this paper on the structure theory of $\mathscr{C}(\mathscr{U} ; V)$ will show that in general this is not the case.

In the next section we characterize the pairs $(\mathscr{U} ; V)$ such that $\mathscr{C}(\mathscr{U} ; V)$ is simple. In Section 3 we investigate the left ideal structure of $\mathscr{C}(\mathscr{U} ; V)$ which results in characterizations of $v$-primitivity for $\mathscr{C}(\mathscr{U} ; V), v=0,1,2$. In Section 4 we study the radicals, $J_{v}(\mathscr{C}(\mathscr{U} ; V)), v=0,1 / 2,1,2$.

\section{Structure of $\mathscr{C}(\mathscr{U} ; \boldsymbol{V})$}

In this section we obtain several properties of the near-ring $\mathscr{C}(\mathscr{U} ; V)$. We first relate the decomposition $V=\sum_{\alpha} \oplus R E_{\alpha} m_{\alpha}$ to the group of units $\mathscr{U}$. Recall from vector space theory that if the $i$ th column of a matrix $A$ is nonzero then there exists a non-singular matrix $P$ such that $A E_{i i}=P E_{i i}$. This establishes the following lemma which suggests that $V$ can be considered as a direct sum of vector spaces of dimension $n$ over $F$ with $\mathscr{U}$ acting on each one naturally.

Lemma 2.1. Let $R=M_{n}(F)$ and let $V$ be a faithful $R$-module. Then $V=\sum_{\alpha} \oplus \mathscr{U}^{0} E_{\alpha} m_{\alpha}$ where $\mathscr{U}^{0}=G L_{n}(F) \cup\{0\}, E_{\alpha} \in\left\{E_{11}, \ldots, E_{n n}\right\}$ and $m_{\alpha} \in V$.

If $V$ is finitely generated over $R$ then the number of nonzero summands in a direct sum decomposition of $V$ into irreducible submodules is unique (see [1], p. 62) so we may call this number $\operatorname{dim}_{R} V$. Otherwise we say $\operatorname{dim}_{R} V=\infty$.

Fundamental to our study of $\mathscr{C}(\mathscr{U} ; V)$ is the orbit structure of the group $V$ by the group of automorphisms $\mathscr{U}$. We have $V=\{0\} \cup\left(\bigcup_{\lambda} \mathscr{U} v_{\lambda}\right)$ where $\{0\} \cup\left\{v_{\lambda}\right\}$ is a complete set of orbit representatives. The set $\left\{v_{\lambda}\right\}$ is called a basis for $V$ over $\mathscr{U}$. For each $v \in V$ we define $\operatorname{stab}(v)=\{A \in \mathscr{U} \mid A v=v\}$. Clearly $\operatorname{stab}(v)$ is a subgroup of $\mathscr{U}$ and for $B \in \mathscr{U}$, $\operatorname{stab} B v=B \operatorname{stab}(v) B^{-1}$. Let $V^{*}=V-\{0\}$ and let $\mathscr{S}=\left\{\operatorname{stab}(v) \mid v \in V^{*}\right\}$. Then $\mathscr{S}$ is partially ordered under set inclusion and we say stab $(v)$ is maximal (minimal) if it is maximal (minimal) in $\mathscr{S}$. The next result due to Betsch (see [6]) points out the importance of the set $\mathscr{S}$ in studying $\mathscr{C}(\mathscr{U} ; V)$. 
Lemma 2.2. Let $x, y \in V$. There exists $f \in \mathscr{C}(\mathscr{U} ; V)$ such that $f(x)=y$ if and only if $\operatorname{stab}(x) \subseteq \operatorname{stab}(y)$.

We consider further the set $\mathscr{S}$. We observe first that for $x \in V, x=x_{a_{1}}+\cdots+x_{\alpha_{1}}$ where the $X_{\alpha_{i}}$ come from different summands of the form $R E_{\alpha} m_{\alpha}$. If $A \in \operatorname{stab}(x)$ then $x=A x=$ $A x_{\alpha_{1}}+\cdots+A x_{\alpha_{i}}$. Hence $A \in \operatorname{stab}\left(x_{\alpha_{i}}\right)$ for each $i$ and so

$$
\operatorname{stab}(x)=\bigcap_{i=1}^{t} \operatorname{stab}\left(x_{\alpha_{i}}\right)
$$

We turn now to a characterization of maximal stabilizers. First consider

$$
x=\left[\begin{array}{c}
1 \\
0 \\
\vdots \\
0
\end{array}\right] m_{\alpha}
$$

Then

$$
\operatorname{stab}(x)=\left\{\left[\begin{array}{ll}
1 & X_{1} \\
0 & X_{2}
\end{array}\right] / X_{1}, X_{2} \text { arbitrary, det } X_{2} \neq 0\right\}
$$

Suppose for $0 \neq y=A_{1} E_{\alpha_{1}} m_{\alpha_{1}}+\cdots+A_{s} E_{\alpha_{s}} m_{\alpha_{s}}, \operatorname{stab}(y) \supseteq \operatorname{stab}(x)$. Let

$$
A_{j} E_{\alpha_{j}} m_{\alpha_{j}}=\left[\begin{array}{c}
b_{1 j} \\
\vdots \\
b_{n j}
\end{array}\right] m_{\alpha_{j}} .
$$

Since

$$
\operatorname{stab}(x) \subseteq \operatorname{stab}(y) \subseteq \operatorname{stab}\left(A_{j} E_{\alpha_{j}} m_{\alpha^{j}}\right)
$$

and since $X_{1}$ is arbitrary in the elements of $\operatorname{stab}(x)$ one finds that $b_{2 j}=\cdots=b_{n j}=0$. Hence

$$
y=\left[\begin{array}{c}
b_{11} \\
0 \\
\vdots \\
0
\end{array}\right] m_{a_{1}}+\cdots+\left[\begin{array}{c}
b_{1 s} \\
0 \\
\vdots \\
0
\end{array}\right] m_{\alpha_{s}}
$$

But then $\operatorname{stab}(y) \subseteq \operatorname{stab}(x)$. Now let $x \in \mathscr{U} E_{\alpha} m_{a}$, say

$$
x=A\left[\begin{array}{c}
1 \\
0 \\
\vdots \\
0
\end{array}\right] m_{a}
$$


and so

$$
\operatorname{stab}(x)=A\left(\operatorname{stab}\left[\begin{array}{c}
1 \\
0 \\
\vdots \\
0
\end{array}\right] \mathrm{m}_{a}\right) \mathrm{A}^{-1}
$$

Hence $\operatorname{stab}(x)$ is maximal. Finally let

$$
y=A_{1} E_{\alpha_{1}} m_{\alpha_{1}}+\cdots+A_{t} E_{\alpha_{t}} m_{\alpha_{t}}
$$

We note that $\operatorname{stab}(y)$ is maximal if and only if $\operatorname{stab}(y)=\operatorname{stab}\left(A_{i} E_{\alpha_{i}} m_{\alpha_{i}}\right)$ for $i=1,2, \ldots, t$. Moreover, for an appropriate $A \in \mathscr{U}$

$$
\operatorname{stab} A y=\operatorname{stab}\left[\begin{array}{c}
1 \\
0 \\
\vdots \\
0
\end{array}\right] m_{\alpha_{i}}=\left\{\left[\begin{array}{cc}
1 & X_{1} \\
0 & X_{2}
\end{array}\right] / X_{1}, X_{2} \text { arbitrary, det } X_{2} \neq 0\right\}
$$

As above this implies

$$
\begin{aligned}
& A A_{j} E_{\alpha_{j}} m_{\alpha_{j}}=\left[\begin{array}{c}
b_{1 j} \\
0 \\
\vdots \\
0
\end{array}\right] m_{\alpha_{j}}, j=1,2, \ldots, t \\
& \text { and so if } A^{-1}=\left(c_{i j}\right), A_{j} E_{\alpha_{j}} m_{\alpha_{j}}=b_{1 j}\left[\begin{array}{c}
c_{11} \\
\vdots \\
c_{n 1}
\end{array}\right] m_{\alpha_{j}} \text {, }
\end{aligned}
$$

i.e., all the $A_{j} E_{a_{j}}$ are in the same 1-dimensional subspace. Conversely if this is the case then a direct calculation shows that

$$
\operatorname{stab}(y)=\operatorname{stab}\left(A_{j} E_{\alpha_{j}} m_{\alpha_{j}}\right), \quad j=1,2, \ldots, t
$$

Hence $\operatorname{stab}(y)$ is maximal.

Theorem 2.3. Let $y \in V, y=A_{1} E_{\alpha_{1}} m_{\alpha_{1}}+\cdots+A_{s} E_{\alpha_{s}} m_{\alpha_{s}} . \operatorname{Stab}(y)$ is maximal if and only if there exists $a_{i} \neq 0$ in $F$ such that

$$
a_{i} A_{i} E_{\alpha_{i}} m_{\alpha_{i}}=A_{1} E_{\alpha_{1}} m_{\alpha_{1}}, \quad i=1,2, \ldots, s
$$

i.e., if and only if $\operatorname{rank}\left[A_{1} E_{\alpha_{1}}, \ldots, A_{s} E_{\alpha_{s}}\right]=1$. 
The next lemma will be used later when studying the $J_{2}$-radical. Since it involves maximal stabilizers we present it here in a general setting.

Lemma 2.4. Let $\mathscr{A} \subseteq$ Aut $G$ and let

$$
\Sigma(g)=\left\{h \in G^{*} \mid \operatorname{stab}(h)=\operatorname{stab}(g)\right\} \cup\{0\}
$$

where $\operatorname{stab}(g)$ is maximal. Then $\Sigma(g)$ is a subgroup of $G$.

Proof. For $h, k \in \Sigma(g)$,

$$
\operatorname{stab}(h-k) \supseteq \operatorname{stab}(h) \cap \operatorname{stab}(k)=\operatorname{stab}(g) .
$$

$\operatorname{But} \operatorname{stab}(g)$ is maximal so $\operatorname{stab}(h-k)=\operatorname{stab}(g)$, hence $h-k \in \Sigma(g)$.

Returning to the partially ordered set $\langle\mathscr{S}, \subseteq\rangle$, let $0 \neq w \in V$,

$$
w=A_{1} E_{\alpha_{1}} m_{\alpha_{1}}+\cdots+A_{s} E_{\alpha_{s}} m_{\alpha_{s}}
$$

and suppose $\operatorname{rank}\left[A_{1} E_{\alpha_{1}}, \ldots, A_{s} E_{\alpha_{s}}\right]=j \leqq n$. Without loss of generality we assume the first $j$ columns are independent. Thus there exists an $A \in \mathscr{U}$ such that

$$
w=A\left[\left[\begin{array}{c}
1 \\
0 \\
\vdots \\
0
\end{array}\right] m_{\alpha_{1}}+\cdots+\left[\begin{array}{c}
0 \\
\vdots \\
0 \\
1 \\
\vdots \\
0
\end{array}\right] m_{\alpha_{j}}\right]+A_{j+1} E_{\alpha_{j+1}}+\cdots+A_{s} E_{\alpha_{s}} m_{\alpha_{s}} .
$$

From this,

$$
\operatorname{stab} A^{-1} w=\left\{\left[\begin{array}{cc}
I_{j} & X_{j 1} \\
0 & X_{j 2}
\end{array}\right] / X_{j 1}, X_{j 2} \text { arbitrary with } \operatorname{det} X_{j 2} \neq 0\right\}
$$

which we henceforth denote by $S_{j}$. This shows that for every nonzero $w$ in $V, \operatorname{stab}(w)$ is conjugate to some $S_{j}$ for a suitable $j$. Thus the $S_{j}$ are canonical representatives of the conjugacy classes in $\mathscr{S}$. In particular we see that $\operatorname{stab}(v)$ is maximal if and only if $\operatorname{stab}(v)$ is conjugate to $S_{1}$. We also find that $\operatorname{stab}(w)$ is minimal if and only if $\operatorname{stab}(w)$ is conjugate to $S_{t}$ where $t=\min \left\{\operatorname{dim}_{R} V, n\right\}$ which in turn is equivalent to

$$
\operatorname{rank}\left[A_{1} E_{\alpha_{1}} m_{\alpha_{1}}, \ldots, A_{s} E_{\alpha_{s}} m_{\alpha_{s}}\right]=t \quad \text { where } \quad w=\sum_{i=1}^{s} A_{i} E_{a_{i}} m_{\alpha_{i}} .
$$

Note that $S_{n}=\{I\}$, the identity matrix. We complete our discussion of $\mathscr{S}$ by showing that $S_{j}$ and $S_{k}$ are not conjugate if $j \neq k$. Thus there will be distinct conjugacy classes if $\operatorname{dim}_{R} V>1$. 
To this end suppose for some $j \neq k, j<k, S_{j}$ is conjugate to $S_{k}$. Observe that all matrices in $S_{k}$ have 1 as an eigenvalue of multiplicity at least $k$ and in $S_{j}$ there are matrices which have 1 as an eigenvalue of multiplicity exactly $j$. Since eigenvalues are preserved under conjugation, $S_{j}$ cannot be conjugate to $S_{k}$.

Summarizing the above, we note that the partially ordered set $\{\mathscr{S}, \subseteq\}$ of stabilizer subgroups has a rather nice structure. Indeed $\langle\mathscr{S}, \subseteq\rangle$ can be thought of as being stratified into $t$ conjugacy layers, $t=\min \left\{\operatorname{dim}_{R} V, n\right\}$, each layer being uniquely determined by a suitable $S_{j}$.

In investigating centralizer near-rings over infinite groups Zeller [8] found the following finiteness condition very useful.

Definition 2.5. ([8]) Let $G$ be a group and $A$ a group of automorphisms of $G$. The pair $(A, G)$ is said to satisfy the finiteness condition (F.C.) if $\operatorname{stab}(x) \subseteq \operatorname{stab}(\alpha x)$ implies $\operatorname{stab}(x)=\operatorname{stab}(\alpha x)$ for $x \in G, \alpha \in A$.

Theorem 2.6. $\mathscr{C}(\mathscr{U} ; V)$ satisfies (F.C.).

Proof. Let $v \in V$ and suppose $\operatorname{stab}(v) \subseteq \operatorname{stab}(A v)$ for some $A \in \mathscr{U}$. From our discussion about $\mathscr{S}$, we know there exists a $B \in \mathscr{U}$ such that stab $B v=S_{k}$ for some $k$ and there are components in $B v$ having column coefficients of the form

$$
\left[\begin{array}{c}
1 \\
0 \\
\vdots \\
0
\end{array}\right],\left[\begin{array}{c}
0 \\
1 \\
0 \\
\vdots \\
0
\end{array}\right], \ldots,\left[\begin{array}{c}
0 \\
\vdots \\
0 \\
1 \\
0 \\
\vdots \\
0
\end{array}\right]
$$

where the last column vector has a 1 in the $k$ th row. Then

$$
\operatorname{stab}(B v) \subseteq \operatorname{stab} B A v=\operatorname{stab} B A B^{-1} B v .
$$

Let $B v=v_{0}$ and $B A B^{-1}=C$. If

$$
C v_{0}=A_{1} E_{\alpha_{1}} m_{\alpha_{1}}+\cdots+A_{t} E_{\alpha_{1}} m_{\alpha_{1}}
$$

then, since $S_{k} \subseteq \operatorname{stab}\left(C v_{0}\right)$, we have

$$
A_{i} E_{\alpha_{i}}=\left[\begin{array}{c}
a_{1 i} \\
\vdots \\
a_{k i} \\
0 \\
\vdots \\
0
\end{array}\right]
$$


Let

$$
C=\left[\begin{array}{ll}
C_{1} & C_{2} \\
C_{3} & C_{4}
\end{array}\right]
$$

where $C_{1}$ is a $k \times k$ matrix. Then because of the form of the above column coefficients in $v_{0}$ and because of the form of the column coefficients in $C v_{0}$ we conclude that $C_{3}=0$. Therefore $C^{-1}$ has the same form and consequently stab $C v_{0}=C S_{k} C^{-1} \subseteq S_{k}$. Hence $\operatorname{stab} C v_{0}=\operatorname{stab} v_{0}$ which in turn gives $\operatorname{stab}(v)=\operatorname{stab}(A v)$ as desired.

Zeller [8] also showed that if $(A, G)$ satisfies (F.C.) and there are at least two conjugacy classes of stabilizers then the centralizer near-ring determined by $(A, G)$ is not simple. From the above theorem and the fact that if $\operatorname{dim}_{R} V>1$ there are distinct conjugacy classes we have the following.

Corollary 2.7. If $\operatorname{dim}_{R} V>1$ then $\mathscr{C}(\mathscr{U} ; V)$ is not simple.

The converse of this corollary is also true.

Theorem 2.8. If $\operatorname{dim}_{R} V=1$ then $\mathscr{C}(\mathscr{U} ; V)$ is simple and in this case $\mathscr{C}(\mathscr{U} ; V)=\mathscr{C}(R ; V)=$ $\operatorname{End}_{R} V \cong F$.

Proof. Since $\operatorname{dim}_{R} V=1, V=\mathscr{U}^{0} E_{\alpha_{1}} m_{\alpha_{1}}=\mathscr{U} E_{\alpha_{1}} m_{\alpha_{1}} \cup\{0\}$. Thus there is one nonzero orbit. From this and the fact that $\mathscr{C}(\mathscr{U} ; V)$ satisfies F.C. we find that every nonzero $f$ in $\mathscr{C}(\mathscr{U} ; V)$ is a bijection, hence $\mathscr{C}(\mathscr{U} ; V)$ is a near-field. Suppose

$$
f\left(E_{a_{1}} m_{\alpha_{1}}\right)=\left[\begin{array}{c}
a_{1} \\
\vdots \\
a_{n}
\end{array}\right] m_{\alpha_{i}} \text { and } E_{a_{i}}=E_{i i}
$$

For $j \neq i, E_{1 j}$ is nilpotent, so $I+E_{i j} \in \mathscr{U}$. Further $\left(I+E_{i j}\right) E_{i i} m_{\alpha_{1}}=E_{i i} m_{\alpha_{1}}$ while

$$
\left(I+E_{i j}\right) f\left(E_{i i} m_{a_{i}}\right)=\left[\begin{array}{c}
a_{1} \\
\vdots \\
a_{i-1} \\
a_{i}+a_{j} \\
a_{i+1} \\
\vdots \\
a_{n}
\end{array}\right]
$$

From this we conclude that

$$
f\left(E_{i i} m_{a_{1}}\right)=\left[\begin{array}{c}
0 \\
\vdots \\
0 \\
a_{i} \\
0 \\
\vdots \\
0
\end{array}\right] m_{a_{1}}
$$


or $f\left(E_{i i} m_{\alpha_{i}}\right)=a_{i} E_{i i} m_{\alpha_{i}}, a_{i} \in F^{*}$. Thus for $A \in \mathscr{U}, f\left(A E_{i i} m_{\alpha_{i}}\right)=a_{i} A E_{i i} m_{\alpha_{1}}$ which implies that $f=\lambda_{a_{i}}$, i.e. $f$ is just left multiplication by $a_{i}$. Hence under the mapping $f \rightarrow \lambda_{a_{i}}$ we have $\mathscr{C}(\mathscr{U} ; V) \cong F$. Thus $\mathscr{C}(\mathscr{U} ; V)$ is simple. Since $\lambda_{a_{i}} \in \operatorname{End}_{R} V$ we have $\mathscr{C}(\mathscr{U} ; V) \subseteq \operatorname{End}_{R} V$. On the other hand since $\mathscr{U} \subseteq R, \mathscr{C}(R ; V) \subseteq \mathscr{C}(\mathscr{U} ; V)$ and clearly $\operatorname{End}_{R} V \subseteq \mathscr{C}(R ; V)$.

Recall that the Kern of a near-ring $N$ is the set

$$
\operatorname{Kern} N=\{a \in N \mid a(b+c)=a b+a c \text { for all } b, c \in N\} .
$$

In the case that $\langle N,+\rangle$ is abelian, $\operatorname{Kern} N$ is a subring of $N$. We conclude this section by characterizing $\operatorname{Kern}(\mathscr{C}(\mathscr{U} ; V))$.

Theorem 2.9. $\operatorname{Kern}(\mathscr{C}(\mathscr{U} ; V))=\operatorname{End}_{R} V=\mathscr{C}(R ; V)$.

Proof. From the generalization of Theorem 1 of [3] as mentioned in the introduction we know $\operatorname{End}_{R} V=\mathscr{C}(R ; V)$ so it remains to verify the first equality. If $\operatorname{dim}_{R} V=1$ then the result follows from the previous theorem. Thus we suppose $\operatorname{dim}_{R} V>1$. Let $v_{i} \in \mathscr{U}^{0} E_{\alpha_{i}} m_{\alpha_{i}}, \quad v_{j} \in \mathscr{U}^{0} E_{\alpha_{j}} m_{\alpha_{j}}, \quad i \neq j$ and let $v=v_{i}+v_{j}$. Then $\operatorname{stab}(v) \subseteq \operatorname{stab}\left(v_{i}\right), \operatorname{stab}(v) \subseteq \operatorname{stab}\left(v_{j}\right)$ so there exists functions $h_{i}, h_{j} \in \mathscr{C}(\mathscr{U} ; V), h_{i}(v)=v_{i}$, $h_{j}(v)=v_{j}$. For $d \in \operatorname{Kern}(\mathscr{C}(\mathscr{U} ; V))$,

$$
d\left(v_{i}+v_{j}\right)=d\left(h_{i}(v)+h_{j}(v)\right)=d\left(h_{i}+h_{j}\right)(v)=\left(d h_{i}+d h_{j}\right)(v)=d\left(v_{i}\right)+d\left(v_{j}\right) .
$$

Now let $v_{i}, v_{j} \in \mathscr{U}^{0} E_{\alpha_{i}} m_{\alpha_{i}}$. Then there exists $w_{j} \in \mathscr{U}^{0} E_{\alpha_{j}} m_{\alpha_{j}} j \neq i$ such that $\operatorname{stab}\left(v_{j}\right)=$ $\operatorname{stab}\left(w_{j}\right)$. Let $w=v_{i}+w_{j}$. As above there exist $g_{i}, g_{j}$ in $\mathscr{C}(\mathscr{U} ; V)$ such that $g_{i}(w)=v_{i}$, $g_{j}(w)=w_{j}$. Since $\operatorname{stab}\left(w_{j}\right)=\operatorname{stab}\left(v_{j}\right)$, there exists $g \in \mathscr{C}(\mathscr{U} ; V)$ such that $g\left(w_{j}\right)=v_{j}$. Hence $\bar{g}_{j}=g g_{j}$ takes $w$ to $v_{j}$. Again if $d \in \operatorname{Kern}(\mathscr{C}(\mathscr{U} ; V))$ then $d\left(v_{i}+v_{j}\right)=d\left(v_{i}\right)+d\left(v_{j}\right)$. This suffices to show $d \in$ End $V$. Since $d \in \mathscr{C}(\mathscr{U} ; V)$ we now have $d \in \operatorname{End}_{\mathscr{U}} V$. The converse is clear so $\operatorname{Kern}(\mathscr{C}(\mathscr{U} ; V))=\operatorname{End}_{\mathscr{U}} V$. Since $R$ is generated by $\mathscr{U}, \operatorname{End}_{R} V=\operatorname{End}_{\mathscr{U}} V$.

\section{Left Ideals in $\mathscr{C}(\mathscr{U} ; V)$}

In this section we examine various left ideals in $\mathscr{C}(\mathscr{U} ; V)$. We determine all minimal left ideals and then use our characterization to show that there are no nonzero nilpotent left ideals in $\mathscr{C}(\mathscr{U} ; V)$. We further use our characterization of minimal left ideals to establish when $\mathscr{C}(\mathscr{U} ; V)$ is $v$-primitive, $v=0,1,2$.

Notation. For the remainder of this paper we use $N$ to denote the near-ring $\mathscr{C}(\mathscr{U} ; V)$.

For an arbitrary centralizer near-ring $\mathscr{C}(A ; G) \equiv M$, let $e_{x}$ denote the idempotent mapping in $M$ which fixes the orbit $A x$ and maps all other orbits to 0 . In [5] it is shown that if $L$ is a minimal left ideal of $M$ then $L \subseteq M e_{x}$ for some $x \in G$, and under certain conditions related to $x$, the left ideal $M e_{x}$ is minimal. Here we find that all minimal left ideals of $N \equiv \mathscr{C}(\mathscr{U} ; V)$ are of the form $N e_{x}$.

We first give an easy but useful technical result.

Lemma 3.1. Let $L$ be a left ideal of $N \equiv \mathscr{C}(\mathscr{U} ; V)$ contained in $N e_{x}$ for some $x \in V$. Let

$$
T(x)=\{v \in V \mid \operatorname{stab}(v) \supseteq \operatorname{stab}(x)\}
$$


and let

$$
L(x)=\{w \in V \mid w=l(x) \text { for some } l \in L\}
$$

Then for each

$$
y \in T(x)-L(x), y+L(x) \subseteq \mathscr{U} y
$$

Proof. We first note that $T(x)$ is a subgroup of $V$. Now let $y \in T(x)-L(x)$ and assume for some $v$ in $L(x)$ that $y+v \notin \mathscr{U} y$. Thus $y, y+v$ are in different orbits so there exists an $f$ in $N$ such that $f(y)=y$ and $f(y+v)=0$. Further there exist $l \in L, g \in N$ such that $l(x)=v$ and $g(x)=y$. Since $L$ is a left ideal of $N, h=f(g+l)-f g \in L$ and so $h(x) \in L(x)$. But

$$
h(x)=f(y+v)-f(y)=-y .
$$

This is a contradiction since $L(x)$ is group and $y \notin L(x)$.

For $x$ in $V . N e_{x}=\operatorname{Ann}\left(1-e_{x}\right)=\operatorname{Ann}(V-\mathscr{U} x)$ so clearly $N e_{x}$ is a left ideal of $N$. Further $\operatorname{Ann} e_{x}=\operatorname{Ann}(x)$ is a left ideal of $N$ with $N e_{x} \oplus \operatorname{Ann} e_{x}=N$, hence $N e_{x}$ is $N$ isomorphic to $N /$ Ann $e_{x}$. Consequently, $N e_{x}$ is a minimal (strictly minimal) left ideal if and only if $\operatorname{Ann}\left(e_{x}\right)$ is a maximal (strictly maximal) left ideal. Further, $N e_{x}$ is strictly minimal if and only if $\operatorname{stab}(x)$ is maximal. For if $\operatorname{stab}(x)$ is maximal this is indeed the case. If $\operatorname{stab}(x)$ is not maximal then $\operatorname{stab}(x) \subsetneq \operatorname{stab}(y)$ for some $y \in V^{*}$. Hence there exists a mapping $f \in N$ defined by $f(x)=y$ and $f(w)=0$ if $w \notin \mathscr{U} x$. But then $f \in N e_{x}$ and $N f$ is an $N$-subgroup of $N e_{x},(0) \subsetneq N f \subsetneq N e_{x}$.

Theorem 3.2. For each $x \in V^{*}, N e_{x}$ is a minimal left ideal.

Proof. Let $L$ be a nonzero left ideal in $N e_{x}$. Hence $L(x) \neq 0$, say $0 \neq y \in L(x)$ where $y=y_{\alpha_{1}}+\cdots+y_{\alpha_{i}}$ with $y_{\alpha_{i}} \neq 0$ for at least one $i$, say $y_{\alpha_{1}}$. Since $\operatorname{stab}\left(y_{\alpha_{1}}\right) \supseteq \operatorname{stab}(y), y_{\alpha_{1}} \in L(x)$. If $\operatorname{stab}(x)$ is maximal then we know $N e_{x}$ is minimal and $L=N e_{x}$. If $\operatorname{stab}(x)$ is not maximal, $x=x_{\beta_{1}}+\cdots x_{\beta_{3}}$ then from Theorem 2.3, there must be at least two non-zero components. For $x_{\beta_{j}}, \beta_{j} \neq \alpha_{1}$, if $x_{\beta_{j}} \notin L(x)$ then since $x_{\beta_{j}} \in T(x)$ we have from Lemma 3.1, $x_{\beta_{j}}+L(x) \subseteq \mathscr{U} x_{\beta_{j}}$. Hence $x_{\beta_{j}}+y_{\alpha_{1}}=A x_{\beta_{j}}$ for some $A \in \mathscr{U}$. But then $(A-I) x_{\beta_{j}}=y_{\alpha_{1}}$. which contradicts the fact that $R E_{\alpha_{1}} m_{\alpha_{1}} \cap R E_{\beta_{j}} m_{\beta_{j}}=(0)$ for $\alpha_{1} \neq \beta_{j}$. Thus we have $x_{\beta_{j}} \in L(x)$ for $\beta_{j} \neq \alpha_{1}$. For $x_{\beta_{j}}$ where $\beta_{j}=\alpha_{1}$ we have $\beta_{i} \neq \beta_{j}$ such that $x_{\beta_{i}} \neq 0$ and $x_{\beta_{i}} \in L(x)$. Therefore as above if $x_{\beta_{j}}\left(\equiv x_{\alpha_{1}}\right) \notin L(x), x_{\beta_{j}}+x_{\beta_{i}}=B x_{\beta_{j}}, B \in \mathscr{U}$, again leading to a contradiction. From this we find that $x_{\beta_{j}} \in L(x)$ for each $\beta_{j}$ and so

$$
x=\sum_{\alpha=1}^{s} x_{\beta_{j}} \in L(x) .
$$

Thus there exists $h$ in $L$ such that $h(x)=x$, i.e., $e_{x} \in L$ and so $L=N e_{x}$.

We now turn to the problem of showing that $\mathscr{C}(\mathscr{U} ; V)$ has no nonzero nilpotent left ideals.

Theorem 3.3. Let $L$ be a left ideal of $N$ containing no nonzero idempotent elements. Then for each $f$ in $L$, for each $x \in V$ if $f(x) \neq 0$, then $\operatorname{stab}(x) \subsetneq \operatorname{stab} f(x)$. 
Proof. We know $\operatorname{stab}(x) \subseteq \operatorname{stab} f(x)$ for each $f \in L$. If the theorem is false, there exists an $f \in L$ and $x \in V^{*}$ such that $\operatorname{stab}(x)=\operatorname{stab}(f(x))$. Let $y=f(x)$. Thus there exists a map $e_{x y}$ in $N$ such that $e_{x y}(y)=x$ and $e_{x y}(w)=0$ for $w \notin \mathscr{U} y$. Since $e_{x y} \in N, e_{x y} f \in L$ so we may assume $f(x)=x$. Similarly $e_{x} f \in L$ so we may also assume that $f(V) \subseteq \mathscr{U}^{0} x$. Let $K=\{v \in V \mid f(v) \in \mathscr{U} x\}$. Then $K \neq \varnothing$ since $\mathscr{U} x \subseteq K$. If $K=\mathscr{U} x$ then $f(v)=v$ for $v \in \mathscr{U} x$ and $f(v)=0$ for $v \notin \mathscr{U} x$; i.e., $f=e_{x}$ which is a contradiction. Thus there exist $v \in K, v \notin \mathscr{U} x$. Thus for some $A \in \mathscr{U}, f(v)=A x$. Let $f_{1}=e_{x}\left(-e_{v}+f\right)+e_{x} e_{v}$ which is in $L$ since $L$ is a left ideal and $f \in L$. Now $f_{1}(x)=x, f_{1}(v)=e_{x}(-v+f(v))=e_{x}(-v+A x)$ and $f_{1}(y)=f(y)$ if $y \notin(\mathscr{U} x \cup \mathscr{U} v)$. Assume $-v+A x \in \mathscr{U} x$. Then $f_{1}(v)=-v+A x$ so $\left(f-f_{1}\right)(v)=A x-$ $(-v+A x)=v$ while $\left(f-f_{1}\right) w=0$ if $w \notin \mathscr{U} v$. Again this is impossible so we have for all $v \in K-x,-v+A x \notin \mathscr{U} x$ where $f(v)=A x$. We now define a new function $h$ as follows. Let $v_{0}$ be arbitrary but fixed in $K-x$. Define $h$ by $h(y)=y$ if $y \in \mathscr{U} x \cup \mathscr{U} v_{0}, h(y)=-y+f(y)$ if $y \in K-\left(\mathscr{U} x \cup \mathscr{U} v_{0}\right)$ and $h(y)=0$ if $y \notin K$. Let $g_{1}=e_{x} h-e_{x}\left(h-f_{1}\right)$ and $g_{2}=e_{x}\left(f_{1}-h\right)+e_{x} h$. Then $g_{1}, g_{2} \in L$. Now

$$
g_{1}(y)=\left\{\begin{array}{l}
0 \text { if } y \notin K \\
y \text { if } y \in \mathscr{U} x \\
0 \text { if } y \in \mathscr{U} v_{0} \\
y \text { if } y \in K-\left(\mathscr{U} x \cup \mathscr{U} v_{0}\right) \text { and }-y \in \mathscr{U} x \\
0 \text { if } y \in K-\left(\mathscr{U} x \cup \mathscr{U} v_{0}\right) \text { and }-y \notin \mathscr{U} x
\end{array}\right.
$$

and $g_{2} g_{1}=e_{x}$ which is again impossible. Thus the result is established.

Theorem 3.4. Let $L$ be a nonzero left ideal of $N$ containing no nonzero idempotent elements. Then there exists some $x \in V^{*}$ such that $L \cap N e_{x} \neq(0)$.

Proof. Let $f$ be nonzero in $L$ with say $f(x)=y \neq 0$. From the previous theorem $\operatorname{stab}(x) \subsetneq \operatorname{stab}(y)$. Since $e_{y} f \in L$ we suppose without loss of generality that $f(V) \subseteq \mathscr{U}^{0} y$. Let $K=\{v \in V \mid f(v) \in \mathscr{U} y\}$. Then $y \notin K$; for if $f(y)=A y$ for some $A \in \mathscr{U}$, we would have $\operatorname{stab}(y) \subsetneq \operatorname{stab} f(y)=\operatorname{stab}(A y)$ which contradicts the finiteness condition of Theorem 2.6. A similar argument shows that $y \notin \mathscr{U} x$. Let $f_{1}=e_{y}\left(-e_{x}+f\right)+e_{y} e_{x}$. Then $f_{1} \in L$ with $f_{1}(x)=$ $e_{y}(-x+y)$ and $f_{1}(w)=f(w)$ for $w \notin \mathscr{U} x$. If $-x+y \in \mathscr{U} y$ then $f_{1}(x)=-x+y$ and consequently $e_{x}=f-f_{1} \in L$ which is a contradiction. Therefore $-x+y \notin \mathscr{U} y$ so $f_{1}(x)=0$. But then $\left(f-f_{1}\right) w=f(w)$ if $w \in \mathscr{U} x$ while $\left(f-f_{1}\right) w=0$ if $w \notin \mathscr{U} x$. Hence $0 \neq f-f_{1}=f e_{x}$ is in $N e_{x} \cap L$.

Corollary 3.5. If $L$ is a nonzero left ideal of $N$ then $L$ contains an idempotent. Further there are no nonzero nilpotent left ideals in $N$.

Proof. Suppose $L$ is a nonzero left ideal that does not contain an idempotent. From the above theorem, $L \cap N e_{x} \neq(0)$ for some $x \in V^{*}$. But for each $x \in V^{*}, N e_{x}$ is a minimal left ideal so that $N e_{x}=L \cap N e_{x} \subseteq L$. This contradiction establishes the desired result.

Corollary 3.6. Let $L$ be a left ideal of $N$. $L$ is a minimal left ideal if and only if $L=N e_{x}$ for some $x \in V^{*}$. 
Proof. If $L=N e_{x}$ then from Theorem $3.2 L$ is minimal. Conversely, from the above corollary $e_{x} \in L$ for some $x \in V^{*}$ and so $N e_{x} \subseteq L$. Since $L$ is minimal $L=N e_{x}$.

We remark that Lemma 3.1 as well as Theorem 3.3 and Theorem 3.4 do not use the structure of $\mathscr{C}(\mathscr{U} ; V)$ in their proofs and therefore are valid in a more general setting. Indeed these results will hold in any centralizer near-ring $\mathscr{C}(A ; G), A \subseteq$ Aut $G$, in which the $N e_{x}$ are minimal, for $x \in G^{*}$ and such that the finiteness condition (F.C.) is satisfied.

We further apply Theorem 3.2 to obtain information about the v-primitivity of $\mathscr{C}(\mathscr{U} ; V), v=0,1,2$. For the necessary definitions and background material on this topic we again refer the reader to Pilz [7].

Theorem 3.7. For $N=\mathscr{C}(\mathscr{U} ; V)$ the following are equivalent:

(i) $N$ is simple,

(ii) $N$ is 2-primitive,

(iii) $N$ is 1-primitive.

Proof. The equivalence of (ii) and (iii) follows from the general results in [7] (p. 104) since $N$ has an identity.

(i) $\rightarrow$ (ii). Since $N$ is simple, from Theorem $2.8, N$ is a field and so is 2-primitive on $\langle N,+\rangle$.

(ii) $\rightarrow$ (i). It is known that when a near-ring $M$ is 2-primitive with a minimal left ideal then all minimal left ideals are $M$-isomorphic [Pilz, p. 130]. In our situation if $N$ is not simple this is impossible. For if $N$ is not simple, $\operatorname{dim}_{R} V \geqq 2$. Thus if $v=E_{\alpha_{1}} m_{\alpha_{1}}$ then $\operatorname{stab}(v)$ is maximal and hence $N e_{v}$ is a strictly minimal left ideal. On the other hand for

$$
w=\left[\begin{array}{c}
1 \\
0 \\
\vdots \\
0
\end{array}\right] m_{\alpha_{1}}+\left[\begin{array}{c}
0 \\
1 \\
0 \\
\vdots \\
0
\end{array}\right] m_{\alpha_{2}}
$$

$\operatorname{stab}(w)$ is not maximal and so as we have seen the minimal left ideal $N e_{w}$ is not strictly minimal. Hence $N e_{v} ¥ N e_{w}$ as $N$-groups.

To complete the characterizations of $v$-primitivity it remains to consider the case for $v=0$. Here the situation is quite different. In fact $\mathscr{C}(\mathscr{U} ; V)$ is always 0 -primitive.

Theorem 3.8. $\mathscr{C}(\mathscr{U} ; V)$ is 0-primitive.

Proof. We separate the proof into two cases depending on $\operatorname{dim}_{R} V$.

Case 1: $\operatorname{dim}_{R} V \geqq n$. As we have seen

$$
v=\left[\begin{array}{c}
1 \\
0 \\
\vdots \\
0
\end{array}\right] m_{\alpha_{1}}+\cdots+\left[\begin{array}{c}
0 \\
\vdots \\
0 \\
1
\end{array}\right] m_{\alpha_{n}}
$$


is such that $\operatorname{stab}(v)=\{I\}$. But then $N e_{v}=V$ is a minimal left ideal, monogenic and clearly the left annihilator of $V$ in $N$ is $\{0\}$. Hence the $N$-module $V$ is of type 0 , i.e., $\mathscr{C}(\mathscr{U} ; V)$ is 0 -primitive in this case.

Case 2: $\operatorname{dim}_{R} V=t<n$. If

$$
x=\left[\begin{array}{c}
1 \\
0 \\
\vdots \\
0
\end{array}\right] m_{1}+\cdots+\left[\begin{array}{c}
0 \\
\vdots \\
0 \\
1 \\
0 \\
\vdots \\
0
\end{array}\right] m_{t}
$$

then we know $\operatorname{stab}(x)=S_{t}$ and $S_{t}$ is minimal in $\mathscr{S}$. Moreover for any $y \in V$ there exists a $B \in \mathscr{U}$ such that $\operatorname{stab}(B y)=S_{k}$ for some $k$ and so $S_{t} \subseteq S_{k}$. Now $N e_{x}$ is a minimal left ideal, and hence an $N$-group of type 0 , clearly monogenic since for each $f \in N e_{x}, f=f e_{x}$. Let $h \in \operatorname{Ann}\left(N e_{x}\right)$ and let $y$ be arbitrary in $V^{*}$. As we showed above, $\operatorname{stab}(x) \subseteq \operatorname{stab}(B y)$ for some $B E \mathscr{U}$ so there exists a $g \in N$ with $g(x)=B y$. But then $0=h\left(g e_{x}\right)$ implies $0=h(B y)=$ $B h(y)$ so $h(y)=0$. Since $y$ was arbitrary $h \equiv 0$. Hence $N$ is 0 -primitive on $N e_{x}$.

\section{Radicals in $\mathscr{C}(\mathscr{U} ; V)$}

In this section we investigate the structure of the various radicals $J_{v}(N), v=0,1 / 2,1,2$ for the near-ring $N=\mathscr{C}(\mathscr{U} ; V)$. For the necessary definitions we again refer to [7]. As in the case of primitivity, since $N$ contains an identity $J_{1}(N)=J_{2}(N)$.

From Theorem 3.2, $N e_{x}$ is a minimal left ideal for each $x \in V^{*}$. Thus Ann $e_{x}$ is a maximal left ideal for $x \in V^{*}$. Therefore

$$
J_{1 / 2}(N)=\cap\{K \mid K \text { is a maximal left ideal of } N\} \subseteq \bigcap_{x \in V^{*}} \text { Ann } e_{x}=\{0\} .
$$

Thus $J_{1 / 2}=(0)$ and since $J_{0}(N) \subseteq J_{1 / 2}(N), J_{0}(N)=(0)$. Of course this latter result was known already since $N$ is 0 -primitive.

It remains to consider $J_{2}(N)$. We first establish some bounds. Let $\mathscr{B}=\left\{x_{\lambda}\right\} \cup\{0\}$ be a basis for $V$ over $\mathscr{U}$. Let $M=\left\{x_{\lambda} \in \mathscr{B} * \mid \operatorname{stab}\left(x_{\lambda}\right)\right.$ is maximal in $\left.\mathscr{S}\right\}$ and let $\bar{M}=\mathscr{B}-M$. For $x_{\lambda} \in M, \operatorname{Ann}\left(x_{\lambda}\right)$ is a strictly maximal left ideal so $J_{2}(N) \subseteq \bigcap_{x_{\lambda} \in M} \operatorname{Ann}\left(x_{\lambda}\right)$. We note that $\bigcap_{x_{\lambda} \in M}$ Ann $x_{\lambda}=N e_{\bar{M}}$ where $e_{\bar{M}}(x)=x$ if $x \in \bar{M}$ and $e_{\bar{M}}(x)=0$ if $x \in M$.

If $L$ is a strictly maximal ideal not of the form Ann $x_{\lambda}$ for $x_{\lambda} \in M$ then for each $x, L$ $+N e_{x}=N$. If for some $x, L \cap N e_{x}=(0)$ then one finds that $L=\operatorname{Ann}(x)$. Since $N e_{x}$ is minimal, $\operatorname{Ann}(x)$ is maximal so $\operatorname{Ann}(x)=L$, a contradiction. Thus for each $x$, $L \cap N e_{x} \neq(0)$ so $N e_{x} \subseteq L$. This also follows from results in [5]. Consequently for every strictly maximal ideal $L$ not of the form Ann $x_{\lambda}$, for $x_{\lambda} \in M$, we have $L \supseteq \sum_{x \in V} N e_{x}$. Further, $N e_{\bar{M}} \supseteq \sum_{\lambda \in \bar{M}} \oplus N e_{x_{1}}$. Since $J_{2}(N)$ is the intersection of all strictly maximal left ideals of $N$ we have $J_{2}(N) \supseteq \sum_{x_{\lambda} \in \bar{M}} \oplus N e_{x_{\lambda}}$. 
Theorem 4.1. $\quad \sum_{x_{\lambda} \in \bar{M}} \oplus N e_{x_{\lambda}} \subseteq J_{2}(N) \subseteq N e_{\bar{M}}$.

Corollary 4.2. If $\bar{M}$ is finite, $J_{2}(N)=N e_{\bar{M}}=\sum_{x_{\lambda} \in \bar{M}} \oplus N e_{x_{\bar{\lambda}}}$.

The left ideal $\sum_{x_{\lambda} \in \bar{M}} \oplus N e_{x_{\lambda}}$ is precisely the collection of functions $f$ in $N e_{\bar{M}}$ with finite support, i.e., $\operatorname{supp}(f)<\infty$ where

$$
\sup (f)=\{x \in V \mid f(x) \neq 0\} \cap \mathscr{B}=\{x \in \mathscr{B} \mid f(x) \neq 0\} .
$$

We now characterize when this set is $J_{2}(N)$.

Theorem 4.3. (i) Let $F$ be an infinite field. Then $J_{2}(N)=\sum x_{\lambda} \in \bar{M} \oplus N e_{x_{\lambda}}$ if and only if $\operatorname{dim}_{R} V \leqq 2$.

(ii) Let $F$ be a finite field. Then $J_{2}(N)=\sum_{x_{\lambda} \in \bar{M}} \oplus N e_{x_{\lambda}}$ if and only if $\operatorname{dim}_{R} V$ is finite.

Proof. (i) If $\operatorname{dim}_{R} V=0$ then $V=(0)$ while if $\operatorname{dim}_{R} V=1, \mathscr{C}(\mathscr{U} ; V)$ is simple so in both of these cases $J_{2}(N)=(0)=\sum_{x_{\lambda} \in \bar{M}} \oplus N e_{x_{\lambda}}$ since $\bar{M}=\{0\}$. Thus suppose $V=$ $R E_{\alpha_{1}} m_{1} \oplus R E_{\alpha_{2}} m_{2}$. From our investigations of the set $\mathscr{S}$ we know that in this case $v \in \vec{M}$ if and only if $\operatorname{stab}(v)$ is conjugate to $S_{2}$. But this means there exists $A \in \mathscr{U}$ such that $\operatorname{stab}(A v)=S_{2}$ and

$$
A v=v_{0}=\left[\begin{array}{c}
1 \\
0 \\
\vdots \\
0
\end{array}\right] m_{1}+\left[\begin{array}{c}
0 \\
1 \\
0 \\
\vdots \\
0
\end{array}\right] m_{2}
$$

This in turn implies that if $\operatorname{stab}(w)$ is not maximal then $w \in \mathscr{U} v_{0}$. Thus $\bar{M}$ has one nonzero element so from Corollary $4.2, J_{2}(N)=\sum_{z_{\lambda} \in \bar{M}} \oplus N e_{x_{\lambda}}$.

Conversely suppose $\operatorname{dim}_{R} V \geqq 3$. For $a \in F^{*}$, let

$$
x_{a}=\left[\begin{array}{c}
1 \\
0 \\
\vdots \\
0
\end{array}\right] m_{1}+\left[\begin{array}{c}
0 \\
1 \\
0 \\
\vdots \\
0
\end{array}\right] m_{2}+\left[\begin{array}{c}
a \\
0 \\
\vdots \\
0
\end{array}\right] m_{3}
$$

We claim $\mathscr{U} x_{a} \neq \mathscr{U} x_{b}$ if $a \neq b$. Otherwise there would exist $A=\left[a_{i j}\right], B=\left[b_{i j}\right]$ in $\mathscr{U}$ such that $A x_{a}=B x_{b}$. Thus

$$
A x_{a}=\left[\begin{array}{c}
a_{11} \\
\vdots \\
a_{n 1}
\end{array}\right] m_{1}+\left[\begin{array}{c}
a_{12} \\
\vdots \\
a_{n 2}
\end{array}\right] m_{2}+\left[\begin{array}{c}
a a_{11} \\
\vdots \\
a a_{n 1}
\end{array}\right] m_{3}=\left[\begin{array}{c}
b_{11} \\
\vdots \\
b_{n 1}
\end{array}\right] m_{1}+\left[\begin{array}{c}
b_{12} \\
\vdots \\
b_{n 2}
\end{array}\right] m_{2}+\left[\begin{array}{c}
b b_{11} \\
\vdots \\
b b_{n 1}
\end{array}\right] m_{3}
$$


From the uniqueness of representation of elements in $V$ we find that $a=b$, a contradiction. From Theorem 2.3, $\operatorname{stab}\left(x_{a}\right)$ is not maximal. We use the $x_{a}, a \in F^{*}$ as part of a basis $\mathscr{B}$ for $V$ over $\mathscr{U}$. Since $F^{*}$ is infinite, so is $\bar{M}$. We define a function $f$ in $N$ as follows. For each $x_{a} \in \bar{M}$ let

$$
f\left(x_{a}\right)=\left[\begin{array}{c}
1 \\
0 \\
\vdots \\
0
\end{array}\right] m_{1}+\left[\begin{array}{c}
0 \\
1 \\
0 \\
\vdots \\
0
\end{array}\right] m_{2} \equiv w_{0}
$$

and let $f(x)=x$ for $x \in \bar{M}-\left\{x_{a}\right\}_{a \in F^{*}}$. Finally define $f(y)=0$ for $y \in M$. Then $f \in N$ and $f(V) \subseteq \mathscr{U} \bar{M}$. Further, since $w_{0} \in \bar{M}, e_{w_{0}} \in J_{2}(N)$ and so $e_{w_{0}} f \in J_{2}(N)$. But supp $e_{w_{0}} f=$ $\left\{x_{a}\right\}_{x \in F^{*}}$ is infinite so $e_{w_{0}} f \in J_{2}(N)-\sum_{x_{\lambda} \in \bar{M}} \oplus N e_{x_{\lambda}}$.

(ii) Let $F$ be a finite field. Then $R$ is finite. If $\operatorname{dim}_{R} V$ is finite then $V$ is finite and the result follows from Corollary 4.2. If $\operatorname{dim}_{R} V$ is not finite then for $j \geqq 3$, the elements $x_{j}=E_{\alpha_{1}} m_{1}+E_{\alpha_{2}} m_{2}+E_{\alpha_{j}} m_{j}$ are in distinct orbits and so can be used as part of a basis. Also $x_{j} \in \bar{M}$ and $\left\{x_{j}\right\}_{j \geqq 3}$ is infinite. As in the first part of the proof one can find a function $0 \neq g \in J_{2}(N)-\sum_{x_{\lambda} \in \bar{M}} \oplus N e_{x_{\lambda}}$.

If $J_{2}(N) \neq \sum_{x_{\lambda} \in \bar{M}} \oplus N e_{x_{\lambda}}$, what can be said about the functions in $J_{2}(N)$ ? We give a partial answer to this question. Thus for the remainder of this section we take $\bar{M}$ to be infinite and $\operatorname{dim}_{R} V \geqq 3$.

Lemma 4.4. Let $L$ be a strictly maximal left ideal of $N$. Either $e_{M}$ or $e_{\bar{M}}$ is in $L$.

Proof. Suppose $e_{M} \notin L$. Since $L$ is strictly maximal, $L+N e_{M}=N$ so there exist $s \in L$, $n \in N$ such that $s+n e_{M}=1$. Let $s_{1}=e_{\bar{M}} s$. Then $s_{1}(V) \subseteq \mathscr{U} \bar{M}$ and since $s(x)=x$ for $x \in \bar{M}$, $s_{1}$ is a nonzero element in $L$. Let $h=e_{\bar{M}}\left(s_{1}+e_{M}\right)-e_{\bar{M}} e_{M}$. Then $h=e_{\bar{M}}\left(s_{1}+e_{M}\right)$ is in $L$ with $h(x)=x$ for $x \in \bar{M}$ while $h(y)=e_{\bar{M}}\left(s_{1}(y)+y\right)$ for $y \in M$. Since $y \in M$, stab(y) is maximal so stab $f(y)=\operatorname{stab}(y)$. From Lemma $2.4, s_{1}(y)+y \in \mathscr{U} M$ so $h(y)=0$. Thus $e_{\bar{M}}=g \in L$.

Theorem 4.5. $J_{2}(N)=\cap\left\{L \mid L\right.$ is a strictly maximal left ideal containing $\left.e_{M}\right\} \cap N e_{\bar{M}}$.

Proof. Let $\Sigma=\left\{L_{\alpha} \mid L_{\alpha}\right.$ is a strictly maximal left ideal $\}$. By Lemma $4.4, \Sigma=\Sigma_{1} \cup \Sigma_{2}$ where $\Sigma_{1} \cap \Sigma_{2}=\varnothing$ and $\Sigma_{1}=\left\{L_{\sigma} \in \Sigma \mid e_{M} \in L_{\alpha}\right\}, \Sigma_{2}=\left\{L_{\sigma} \in \Sigma \mid e_{\bar{M}} \in L_{\sigma}\right\}$. By definition $J_{2}(N)=$ $\bigcap_{\sigma \in \Sigma} L_{\sigma}$ and since $N e_{\bar{M}} \supseteq J_{2}(N)$ we have $J_{2}(N)=\bigcap_{\sigma \in \Sigma} L_{\sigma} \cap N e_{\bar{M}}$. For $L_{\sigma} \in \Sigma_{2}, N e_{\bar{M}} \subseteq L_{\sigma}$ so $N e_{\bar{M}}=L_{\sigma} \cap N e_{\bar{M}}$. Thus

$$
J_{2}(N)=\bigcap_{\sigma \in \Sigma_{1}} L_{\sigma} \cap\left(\bigcap_{\sigma \in \Sigma_{2}} L_{\sigma}\right) \cap N e_{\bar{M}}=\bigcap_{\alpha \in \Sigma_{1}} L_{\alpha} \cap N e_{\bar{M}}
$$

as desired.

Let $f \in N$. We define the rank of $f$ to be the cardinality of the set $f(V) \cap \mathscr{B} *$ where $\mathscr{B}$ is a basis for $V$. 
Theorem 4.6. $J_{2}(\dot{N}) \supseteq\{f \in N \mid \operatorname{supp}(f) \subseteq \bar{M}$ and rank $f$ is finite $\}$.

Proof. Let $f \in N e_{\bar{M}}$ such that $f$ has finite rank. Let $x_{\lambda_{1}}, \ldots, x_{\lambda_{k}}, x_{\lambda_{k+1}}, \ldots, x_{\lambda_{t}}$, be the basis elements in $f(V)$ where $x_{\lambda_{i}} \in M, i=1,2, \ldots, k$ and $x_{\lambda_{j}}, j=k+1, \ldots, t$ are in $\bar{M}$. Thus $f$ can be represented as $f=f_{1}+f_{2}$ where $f_{1}=\sum_{i=1}^{k} e_{x_{\lambda}} f$ and $f_{2}=\sum_{j=k+1}^{t} e_{x_{\lambda_{j}}} f$. Since $e_{x_{\lambda_{3}}} \in J_{2}(N), j=k+1, \ldots, t$ so does $f_{2}$. Hence if $f \notin J_{2}(N)$ then $f_{1} \notin J_{2}(N)$. This in turn implies that one of the summands, say without loss of generality $e_{x_{\lambda_{1}}} f$, is not in $J_{2}(N)$. Let $g=e_{x_{\lambda_{1}}} f$ and let supp $g=\bar{M}_{1}$. If $\bar{M}_{1}$ is finite then $g \in J_{2}(N)$ so we assume $\bar{M}_{1}$ is infinite. Since $g \in N e_{\bar{M}}$ but is not in $J_{2}(N)$ there exists a strictly maximal left ideal $L$ in $\Sigma_{1}$ such that $g \notin L$. Hence $L+N g=N$ and so there exist $s \in L, n \in N$ such that $s+n g=1$. Therefore $s(x)=x$ for $x \notin \bar{M}_{1}$ and for $x \in \bar{M}_{1} s(x)=x-n\left(A x_{\lambda_{1}}\right)$ for some $A \in \mathscr{U}$. Clearly $n\left(A x_{\lambda_{1}}\right) \neq 0$. Thus $n\left(A x_{\lambda_{1}}\right) \in \mathscr{U} M$ since $x_{\lambda_{1}} \in M$. Let $h=e_{M}\left(e_{\bar{M}_{1}}-s\right)-e_{M} e_{\bar{M}_{1}}=e_{M}\left(e_{\bar{M}_{1}}-s\right)$ since $\bar{M}_{1} \subseteq \bar{M}$. Then $h \in L$ and for $x \in \bar{M}_{1}, h(x)=e_{M}(x-s(x))=x-s(x)$ since $n\left(A x_{1}\right) \in \mathscr{U} M$ while for $x \notin \bar{M}_{1}$

$$
h(x)=e_{M}(-x)=\left\{\begin{array}{rl}
-x & \text { if } x \in M \\
0 & \text { if } x \in \bar{M}-\bar{M}_{1}
\end{array} .\right.
$$

Since $h \in L, h_{1}+s$ is also in $L$ and

$$
h_{1}(x)=\left\{\begin{array}{l}
x \text { if } x \in \bar{M}_{1} \\
0 \text { if } x \in M \\
x \text { if } x \in \bar{M}-\bar{M}_{1} .
\end{array}\right.
$$

Therefore $e_{\bar{M}}=h_{1} \in L$ which is a contradiction. Consequently $f \in J_{2}(N)$.

In a similar manner we now show that $J_{2}(N)$ contains all functions with support in $\vec{M}$ and range in $\mathscr{U} M \cup\{0\}$.

Theorem 4.7. $J_{2}(N) \supseteq\left\{f \in N \mid f \in N e_{\bar{M}}\right.$ and $\left.f(\bar{M}) \subseteq \mathscr{U} M \cup\{0\}\right\}$.

Proof. Let $f \in N e_{\bar{M}}$ with $f(\bar{M}) \subseteq \mathscr{U} M \cup\{0\}$. Further let $\bar{M}_{1}=\operatorname{supp} f$. If $f \notin J_{2}(N)$ then as in Theorem 4.6 there exists a strictly maximal left ideal $L$ with $e_{M} \in L$ and $s \in L, n \in N$ with $s+n f=1$. Since $f(x) \in \mathscr{U} M$ so does $n f(x)$ for all $x \in \bar{M}_{1}$. Now $h=e_{M}\left(e_{\bar{M}_{1}}-s\right)-e_{M} e_{\bar{M}_{1}}=$ $e_{M}\left(e_{\bar{M}_{1}}-s\right)$ is in $L$. As above $h_{1}=h+s$ is in $L$ and $h_{1}=e_{\bar{M}}$ a contradiction. Thus $f \in J_{2}(N)$.

The problem of characterizing the elements in $J_{2}(N)$ remains open. That the above two results do not give this characterization is pointed out in the following example in which we give a function $f$ in $J_{2}(N)$ with $f(\bar{M}) \subseteq \mathscr{U} \bar{M}$ and $f$ is not of finite rank.

Example 4.8. Let $\operatorname{dim}_{R} V$ be at least 3 and $F$ an infinite field. Let

$$
x_{a}=\left[\begin{array}{c}
1 \\
0 \\
\vdots \\
0
\end{array}\right] m_{1}+\left[\begin{array}{c}
0 \\
1 \\
0 \\
\vdots \\
0
\end{array}\right] m_{2}+\left[\begin{array}{c}
a \\
0 \\
\vdots \\
0
\end{array}\right] m_{3}
$$


$a \in F^{*}$ as in Theorem 4.3. Define $f_{1}$ by

$$
f_{1}\left(x_{a}\right)=\left[\begin{array}{c}
1 \\
0 \\
\vdots \\
0
\end{array}\right] m_{1}+\left[\begin{array}{c}
0 \\
1 \\
0 \\
\vdots \\
0
\end{array}\right] m_{2}
$$

and $f_{1}$ to be zero on the other basis elements. Since $f_{1}$ is of finite rank, $f_{1} \in J_{2}(N)$. Define $f_{2}$ by

$$
f_{2}\left(x_{a}\right)=\left[\begin{array}{c}
a \\
0 \\
\vdots \\
0
\end{array}\right] m_{3}
$$

and $f_{2}$ to be zero on the other basis elements. Since $f_{2}(\bar{M}) \subseteq \mathscr{U} M \cup\{0\}, f_{2} \in J_{2}(N)$. Hence $f=f_{1}+f_{2} \in J_{2}(N)$ where $f$ is the identity on $\left\{x_{a}\right\}_{a \in F^{*}}$ and $f$ is zero on the other basis elements.

We conclude with a definite result for the situation in which $\operatorname{dim}_{R} V$ is finite.

Theorem 4.9. Let $\operatorname{dim}_{R} V$ be finite and let $f \in N e_{\bar{M}}$. Then $f \in J_{2}(N)$ if and only if $f$ is the sum of rank 1 functions.

Proof. Suppose $f=\sum_{j=1}^{n} f_{j}$ where $f_{j}$ is a rank 1 function. Since each $f_{j}$ is in $J_{2}(N)$, so is $f$. Conversely let $f \in J_{2}(N)$ and let $\pi_{i}$ be the ith projection map $i=1,2, \ldots, t$ where $t=\operatorname{dim}_{R} V$. Since $\pi_{i} \in \operatorname{End}_{R} V, \pi_{i} \in N$ so $\pi_{i} f \in J_{2}(N)$ and $\pi_{i} f$ is of rank 1. But $f=\sum_{i=1}^{t} \pi_{i} f$ so the proof is complete.

Acknowledgement. The first author would like to acknowledge financial support from Teesside Polytechnic where he was visiting when this work was done.

\section{REFERENCES}

1. N. JACosson, Structure of Rings (Amer. Math. Soc. Coll. Publ. Vol. 37, Providence, RI, 1964).

2. C. J. Maxson and K. C. SMrth, The centralizer of a group automorphism, J. Algebra 54 (1978), 27-41.

3. C. J. Maxson and K. C. Sмiтr, Simple near-ring centralizers of finite rings, Proc. Amer. Math. Soc. 75 (1979), 8-12.

4. C. J. Maxson and K. C. Sмrth, The centralizer of a set of group automorphisms, Comm. in Alg. 8 (1980), 211-230.

5. C. J. Maxson and K. C. SMith, Centralizer near-rings: Left ideals and 0-primitivity, Proc. Royal Irish Acad. 81 (1981), 187-199.

6. J. D. P. Meldrum and A. Oswald, Near-rings of mappings, Proc. Royal Soc. Edinburgh 83A (1978), 213-223. 
7. G. Pilz, Near-rings (North-Holland, New York, 1977).

8. M. ZelleR, Centralizer near-rings on infinite groups (Ph.D. Dissertation, Texas A\&M University, College Station, 1980).

Department of Mathematics

TEXAS A\&M UNIVERSITY

College Station, TX 77843

USA
Department of Mathematics and Statistics Teesside Polytechnic

Borough Road

Middlesbrough

Cleveland TS1 3BA

UK 